\title{
Factores de riesgo suicida en el anciano
}

\author{
Suicide risk factors among the elderly
}

Sergio Andrés Pérez Barrero ${ }^{1}$

${ }^{1}$ Sección de Suicidiologia, Sociedad Cubana de

Abstract The author offers a brief overview of suicide risk factors among the elderly such as depression, all manner of abuse of the elderly, as well as medical, psychological and social risk factors, etc. By way of conclusion, a practical guide to evaluate suicide risk among the elderly is provided.

Key words Suicide risk factors, Elderly people, Practical guide
Resumen El autor expone brevemente los factores de riesgo suicida en la vejez, entre los que se mencionan la depresión, el abuso en cualquiera de sus formas de presentación, los factores médicos, psicológicos, sociales, etc. Finalmente se ofrece una guía práctica para evaluar el riesgo de suicidio en el anciano.

Palabras claves Factores de riesgo suicida, anciano, guía práctica 


\section{Introducción}

A pesar de la presión popular sobre el suicidio en la juventud y las numerosas investigaciones en torno a este fenómeno, el suicidio entre los ancianos constituye una significativa causa de muerte. En la medida en que las personas mayores conforman el segmento de más rápido crecimiento de la población, el número absoluto de sus suicidios continuará incrementándose y se pronostica que para el 2030 será el doble, por lo que se hace necesario profundizar en el conocimiento de los factores de riesgo en la vejez para atenuar en cierta medida dicha predicción ${ }^{1,2}$

El presente artículo hace énfasis en los factores de riesgo, teniendo en cuenta el conocimiento de la literatura internacional pero, sobre todo, la experiencia del autor en el estudio de la prevención del suicidio.

\section{Desarrollo}

Es conocido que la conducta suicida en el anciano tiene los siguientes rasgos distintivos: (1) ellos realizan menos intentos de suicidio que los jóvenes. Por cada anciano suicida lo han intentado cuatro, mientras que por cada joven suicida, lo han intentado doscientos. En la población en general, por cada suicidio ocurren entre 15 a 20 intentos de suicidio, proporción que es mayor que la observada en la vejez; (2) utilizan métodos mortales (el 85\% de los suicidios en los hombres ancianos es por ahorcamiento, armas de fuego y precipitación de lugares elevados); (3) reflejan menos señales de aviso y estas son más difíciles de detectar; (4) dichos actos suicidas no son impulsivos, sino meditados, realizados después de un detenido proceso de reflexión; (5) pueden asumir la forma de suicidios pasivos (no ingerir alimentos, para dejarse morir).

Es muy importante que los médicos de familia, los geriatras y los familiares conozcan las particularidades de la comunicación suicida en la vejez, por que tiene características distintivas que la diferencia de las que se observan en la infancia, la adolescencia y la adultez. Es habitual que las personas mayores expresen sus intenciones suicidas refiriendo que son una carga para los demás, y que no desean seguir siéndolo y que los demás estarían mejor si ellos estuvieran muertos. Consideran que ya han vivido demasiado, que no tiene sentido que continúen viviendo y que no desean darle más trabajo a los seres queridos.

Otros componentes del comportamiento suicida frecuentes en otras etapas de la vida como las amenazas, los gestos, los intentos de suicidio y el suicidio accidental son raros en la vejez, mientras que son muy frecuentes y habituales los suicidios frustrados considerados como tal, o sea, aquellos actos suicidas que no ocasionan la muerte debido a la concurrencia de circunstancias fortuitas, casuales e imprevistas. Junto a los suicidios intencionales son las manifestaciones mas frecuentes de la comunicación suicida en los ancianos ${ }^{3,4}$. Una manera de comunicar los ancianos sus intenciones suicidas consiste en asumir formas de presentación no suicida como encamarse y dejar de ingerir alimentos, transgredir regimenes terapéuticos como una forma velada de producirse la muerte por las complicaciones que estas transgresiones provocan en la salud o autoexcluirse, no inyectarse insulina en el caso de diabéticos insulinodependientes, no tomar los antihipertensivos aquellos que padecen de hipertensión arterial severa, o autoexcluirse como es el caso de los ancianos inuits quienes deciden alejarse de sus seres queridos para morir de frío y no seguir consumiendo sin aportar a la familiaa ${ }^{5-8}$.

Por lo general, los ancianos para cometer el suicidio utilizan métodos de fácil disponibilidad que conllevan elevada letalidad entre los que sobresalen el ahorcamiento, la precipitación, el disparo de arma de fuego, la ingestión de venenos agrícolas y la intoxicación con monóxido de carbono. Son suicidios que se realizan con aparente lógica y esta característica condiciona que sean calificados como "suicidios racionales", término utilizado para definir aquellos que ocurren en ausencia de una enfermedad mental, como supuesta expresión de la libertad del sujeto para elegir su propia muerte, sobre todo en aquellas situaciones en las que la vida no ofrece oportunidad alguna. Así, se supone que existe elevada intencionalidad y motivaciones racionales que justifican dicho acto, pero que para un observador entrenado hay un trasfondo depresivo en la mayoría de los casos.

No son infrecuentes los pactos suicidas entre cónyuges añosos que están deprimidos o situaciones de homicidio-suicidio, en los que el perpetrador habitualmente ha presentado manifestaciones depresivas relevantes.

A continuación se relacionan las formas mas frecuentes de la comunicación suicida directa verbal que suelen observarse en los ancianos. Estas son todas expresiones que se oye en los estudios de autopsia psicológica:

- Había dicho que tenía ideas de matarse.

- Había dicho que tenía deseos de morirse

. Había dicho que no quería seguir viviendo 
- Había dicho que los demás se sentirían mejor si el no existiera

- Había dicho que la vida no era digna de ser vivida

- Había dicho que quería matarse

- Había dicho que era mejor estar muerto que vivo

- Había dicho que quería terminar de una vez por todas

- Había dicho que no quería seguir siendo una carga para los demás

- Había dicho que la vida no valía la pena vivirla

- Había dicho que le gustaría dormirse y no despertar nunca más

- Había dicho que estaba cansado de la vida

- Había dicho que ya no tendrían que seguir soportándolo: usual en las crisis suicidas que se dan en el contexto de conflictos familiares.

- Había amenazado con suicidarse

- Había expresado deseos de descansar de todo

- Había dicho que no deseaba darle más trabajo a sus

- Había manifestado no hacerle falta a otros

- Había considerado que la ayuda era inútil y una perdida de tiempo.

En cuanto a la comunicación suicida directa no verbal en los ancianos se pueden observar las siguientes conductas:

- Había estado buscando los medios para cometer suicidio

- Había estado poniendo sus asuntos en orden

- Había estado regalando sus posesiones más valiosas

. Después de una fase de agitación había mostrado el llamado período de calma consistente en cese de la angustia, sensación de paz y tranquilidad internas todo lo que debe interpretarse como signos de grave peligro suicida, pues se ha resuelto el conflicto entre los deseos de vivir y los deseos de morir a favor de estos últimos (la calma antes de la tormenta

- Ha mantenido escondido el futuro método para lograr realizar el suicidio (llevar consigo la soga, adquirir un arma de fuego, etc.)

- Se ha dirigido hacia el lugar elegido para realizar el acto suicida

- Había escrito notas diciendo lo que haría o dejado notas de despedida

- Había insistido en hacer testamento sin tener manifestaciones de enfermedad física terminal que pudiera justificarlo

- Había asumido la forma de un suicidio pasivo, (permanecen en el lecho y dejan de ingerir alimentos o los rechazan, manifestando que los dejen morir en paz y tranquilidad)

- Ha presentado una actitud muy demandante de atención con marcado comportamiento histriónico, como una manera desesperada y primitiva de pedir auxilio

. Había realizado un suicidio frustrado.

El envejecimiento trae consigo el abandono de la profesión o de otros objetivos, la reducción del vigor físico, la modificación de los placeres sensuales y una conciencia de muerte desconocida en etapas previas de la vida. El anciano no siempre aporta síntomas porque teme que le puedan diagnosticas una enfermedad grave o porque asuman sus quejas como una parte del proceso del envejecimiento. Entre los problemas físicos que el anciano debe afrontar se encuentran la patología artrítica, que afecta su locomoción; las enfermedades cardiovasculares que limitan el ejercicio físico; las enfermedades neurológicas que bloquean las funciones intelectuales y el cáncer que ocasiona dolor y dependencia ${ }^{9-12}$.

Los problemas emocionales que padece el anciano incluyen la depresión, que es la más común de las enfermedades mentales en la vejez y el mejor predictor del suicidio del anciano. Se considera que dos tercios de los suicidios geriátricos están asociados con la depresión que puede adquirir las siguientes formas de presentación:

Depresión que se presenta como un aparente envejecimiento normal - En este caso el anciano muestra disminución del interés por las cosas que habitualmente lo despertaban, de la vitalidad, de la voluntad; ten-dencia a revivir el pasado, pérdida de peso, trastornos del sueño, algunas quejas por falta de memoria, tiende al aislamiento y permanece la mayor parte del tiempo en su habitación. (Para muchos este cuadro es propio de la vejez y no una depresión tratable).

Depresión que se presenta como un envejecimiento anormal - En el anciano aparecen diversos grados de desorientación en lugar, en tiempo y con respecto a sí mismo y a los demás: confunde a las personas conocidas, es incapaz de reconocer lugares; aparecen deterioro de sus habilidades y costumbres, relajación esfinteriana, esto es, se orina y defeca sin control alguno, trastornos de la marcha que hacen pensar en una enfermedad cerebrovascular, trastornos de conducta como negarse a ingerir alimentos, labilidad afectiva, etc. (Para muchos este cuadro es propio de una demencia con carácter irreversible y no una depresión tratable).

Depresión que se presenta como una enfermedad física, somática u orgánica - El anciano se 
queja de múltiples síntomas físicos, como dolores de espalda, en las piernas, en el pecho, cefaleas. Puede quejarse también de molestias digestivas como digestión lenta, acidez, plenitud estomacal sin haber ingerido alimentos que lo justifiquen; tiende a tomar -la-xantes, antiácidos y otros medicamentos para sus molestias gastrointestinales; refiere pérdida de la sensación del gusto, falta de apetito y disminución del peso, problemas cardiovas-cula-res como palpitaciones, opresión, falta de aire, etcétera. (Para muchos este cuadro es propio de alguna enfermedad del cuerpo y no una depresión tratable).

Depresión que se presenta como una enfermedad psiquiátrica no depresiva - El anciano manifiesta que lo persiguen, que lo están velando, que lo quieren matar, que la policía lo va a venir a buscar para llevarlo preso y estas ideas forman parte de la constelación paranoide. Sin embargo, cuando se le pregunta por qué le está sucediendo esto, aflora el componente delirante depresivo al responder que eso ocurre porque él "es el causante de todos los daños del universo, que él se lo tiene merecido para expiar todas las culpas por todo lo abominable de su pasado".

Depresión que se presenta como enfermedad psiquiátrica depresiva - El anciano manifiesta tristeza, pocos deseos de hacer las cosas, ideas suicidas persistentes, ideas de minusvalía y autorreproche, pérdida de autoestima, lentitud psíquica y motora, trastornos del sueño y el apetito, descuido del aseo personal y angustia ${ }^{1-3}$.

A la depresión en la vejez se pueden añadir otros factores como las propias presiones sociales derivadas de la jubilación, dependencia, muerte de seres queridos, familiares o amigos, la pérdida de la seguridad económica que incrementan el riesgo de suicidio. Como se hace evidente, existen suficientes condiciones inherentes a la vejez que son un caldo de cultivo adecuado para que se manifieste la conducta autodestructiva ${ }^{2}$

El intento de suicidio en los ancianos es un serio problema, pues en la generalidad de los casos se trata de verdaderos suicidios frustrados por su elevado grado de premeditación, por los métodos mortales que se utilizan, por la coexistencia de enfermedades físicas principalmente las enfermedades pulmonares obstructivas crónicas o los trastornos gastrointestinales y músculo esqueléticos, así como determinados síntomas físicos intratables, en especial, el dolor o la falta de aire.

Nunca se debe olvidar que los síntomas somáticos pueden ser un signo de depresión, pues los ancianos deprimidos tienen la tendencia a res- tar importancia a la tristeza, a la que muchas veces consideran una parte consustancial de la vejez, quejándose principalmente de los síntomas físicos: de que "algo no anda bien en la salud", considerándose ellos mismos como enfermos físicos, lo que les hace frecuentar internistas o médicos de atención primaria, aplazando el inicio de un tratamiento antidepresivo eficaz y específico. Los médicos que no están familiarizados con esta condición no podrán realizar el diagnóstico precoz y el riesgo de suicidio puede emerger durante la supuesta enfermedad física, pues al no obtener mejoría alguna con los tratamientos impuestos, el anciano comienza a pensar que su enfermedad es maligna, que le ocasionará una prolongada agonía y opta por el suicidio para evitar los sufrimientos que él avizora.

Las señales biológicas de la depresión como el insomnio, pérdida de peso, apetito y energía, pirosis, acidez, digestiones lentas, constipación, palpitaciones, dolores en el pecho, mialgias, artralgias, lumbalgias y dolores de espaldas son atribuidas por el anciano, como se ha señalado con antelación, a una enfermedad física y si el médico no está familiarizado con esta forma de presentación de los trastornos del humor, puede ocurrir que una depresión no suicida se convierta en una depresión suicida al no imponerse el tratamiento antidepresivo específico ${ }^{3,4,13-16}$

Otro factor de riesgo suicida en la vejez es el maltrato a que son sometidos muchos ancianos, existiendo una estrecha relación entre la pobre salud física, la conducta suicida y las situaciones de maltrato. Por definición se considera el maltrato al anciano como "la acción única o repetida, o la falta de una respuesta apropiada, que ocurre dentro de cualquier relación donde exista una expectativa de confianza y la cual produzca daño o angustia a una persona anciana". Puede realizarse de diversas maneras, entre las que sobresalen el maltrato físico, el psicológico, el sexual, el económico, el emocional o por omisión, descuido o negligencia en su cuidado. El maltrato al anciano es perpetrado por los familiares y los cuidadores que tienen una historia de violencia y conducta antisocial. La enfermedad mental y el abuso de sustancias predisponen a los familiares a maltratar sus ancianos, lo cual se hace fácilmente reconocible para un clínico alerta, pues se pueden presentar lesiones en diversos estadios de evolución o cuando la explicación de los daños evidenciables es vaga o imprecisa. Otras manifestaciones que pueden hacer sospechar la existencia del maltrato a un anciano son las siguientes: (1) demora entre la ocurrencia del daño o el inicio de la enfer- 
medad y la búsqueda de atención médica; (2) diferencias entre las historias aportadas por el anciano y los posibles maltratadores o victimarios; (3) frecuentes visitas a los médicos por exacerbaciones de enfermedades crónicas a pesar de tener un plan terapéutico eficaz; (4) angustia, confusión, depresión, ideas suicidas e intentos de suicidio pueden ser las respuestas de un anciano a los maltratos físicos o psicológicos.

A partir de lo expuesto se puede decir, en síntesis, que los factores de riesgo para el suicidio de anciano se dividen en:

Factores médicos - (a) enfermedades crónicas, terminales, dolorosas, invalidantes, incapacitantes, como la demencia senil tipo Alzheimer, la enfermedad de Parkinson, las neoplasias, la diabetes mellitus complicada con retinopatía o polineuropatía, la insuficiencia cardiaca congestiva, la enfermedad pulmonar obstructiva crónica; (b) la hospitalización periódica del anciano, así como ser sometido a intervenciones quirúrgicas frecuentes, principalmente del aparato genitourinario o gastrointestinal; (c) los tratamientos prodepresivos muy utilizados para contrarrestar enfermedades que frecuentemente padece el anciano (digitálicos, propanolol, indometacina, metildopa, etc.) (d) las enfermedades prodepresivas como la arteriosclerosis, las demencias, el carcinoma de cabeza de páncreas y otras.

Factores psiquiátricos - Se incluyen las depresiones de cualquier naturaleza, el abuso de alcohol y de drogas, los trastornos crónicos del sueño, las psicosis delirantes paranoides con gran agitación y desconfianza, la confusión mental.

Factores psicológicos - Los sentimientos de soledad e inutilidad, inactividad, aburrimientos, falta de proyectos vitales y tendencia a revivir el pasado.

Factores familiares - (a) pérdida de seres queridos por muertes naturales o por suicidio; (b) la viudez durante el primer año es un momento crítico para el anciano, durante el cual puede ocurrir la llamada autodestrucción pasiva, en la que el evento vital desencadena una depresión que altera el sistema inmunológico lo que facilita la aparición de enfermedades físicas, principalmente las infecciosas que pueden dar al traste con la vida del anciano; (c) el caso del abuelo "ping pong" que se produce cuando se condena al anciano a la migración forzosa al ser trasladado de un domicilio a otro a conveniencia de los familiares, en detrimento de su comodidad, privacidad y estabilidad; (d) el ingreso en un hogar de ancianos en su etapa de adaptación o cuando se realiza en contra de la voluntad del anciano suele reactivar situaciones de desamparo previas que pueden precipitar un acto suicida.

Factores socio-ambientales - (a) la jubilación; (b) el aislamiento social; (c) la actitud hostil, peyorativa o despreciativa de la sociedad hacia sus ancianos; (d) la competencia de las generaciones más jóvenes; (e) la pérdida de prestigio ${ }^{17-23}$

Para concluir este ensayo, se propone una Guía Practica que ha sido validada por su uso desde 1995 en la atención primaria de salud en Cuba, formando parte de los instrumentos del Programa Nacional de Prevención del Suicidio desde 2005. El objetivo cardinal de dicha Guía es dotar al medico de atención primaria de un instrumento de fácil aplicación para reconocer cuando un anciano presenta una situación de crisis suicida y derivarlo oportunamente a los servicios especializados de salud mental para que su evaluación precoz. Su utilización continuada, contribuye a que el medico de atención primaria se apropie de las manifestaciones clínicas habituales en esta etapa de la vida que requieren referencia oportuna al psiquiatra para evaluar la peligrosidad suicida oportunamente (Cuadro 1).

Por la experiencia y observación, si la suma de la puntuación es mayor de 9, el anciano debe ser remitido a una unidad psiquiátrica para ser examinado por el personal especializado ${ }^{24}$.

Los factores de riesgo suicida que se pueden hacer evidentes en la vejez y que al conjugarse varios de ellos, se incrementa el riesgo que el anciano termine su vida por suicidio, aquí descritos coinciden con lo que se conoce hoy en la literatura internacional y en ámbito de la Organización Mundial de la Salud ${ }^{10-16}$ 
Cuadro 1. Factores asociados al suicidio de ancianos

\begin{tabular}{|l|c|}
\hline \multicolumn{1}{|c|}{ Item } & Puntuación \\
\hline 1.Actitud poco cooperadora del anciano en la entrevista & 2 puntos \\
\hline $\begin{array}{l}\text { 2. Padecer una enfermedad física que ha requerido ingresos frecuentes y tratamientos } \\
\text { prolongados }\end{array}$ & 2 puntos \\
\hline 3. Padecer una enfermedad mental & 3 puntos \\
\hline 4. Tener antecedentes personales de intento suicida & 3 puntos \\
\hline $\begin{array}{l}\text { 5. Poseer antecedentes familiares de conducta suicida } \\
\text { 6. El anciano vive solo }\end{array}$ & 1 punto \\
\hline $\begin{array}{l}\text { 7. Expresar deseos de descansar de todo, no dar más trabajo a otros y tener el } \\
\text { sentimiento de no hacer falta a otros }\end{array}$ & 4 puntos \\
\hline $\begin{array}{l}\text { 8. Manifestar ideas o plan suicida } \\
\text { 9. Manifestar cambios de conducta en forma de aislamiento, agresividad, agitación, } \\
\text { llanto frecuente de pocos días de evolución }\end{array}$ & 3 puntos \\
\hline $\begin{array}{l}\text { 10. Negarse a recibir ayuda por considerar que es inútil, que es perder el tiempo, a pesar } \\
\text { de mantener una actitud adecuada en la entrevista. }\end{array}$ & 5 puntos \\
\hline
\end{tabular}

\section{Referencias}

1. Pérez BSA. El suicidio comportamiento y prevención. Santiago de Cuba: Editorial Oriente; 1997.

2. Pérez BSA. Psicoterapia para aprender a vivir. Santiago de Cuba: Editorial Oriente; 2001.

3. Pérez BSA. Lo que usted debiera saber sobre... Mexico: Editorial Imagen; 1999.

4. Pérez BSA Psicoterapia del comportamiento suicida. Habana: Editorial del Hosp. Psiq. de La Habana; 2001.

5. Bron B. Suicidal risk in endogenous, neurotic and reactive depression in advanced age Schweiz. ArchNeurol, Psychiatr 1990; 14(3):229-253.

6. Comuel Y, Rotenberg M, Caine E. Completed suicide at age 50 and over. Crisis 1990; 38(6):640-644.

7. De las Heras F, Dueñas M. Etiopatogenia e incidencia del suicidio entre los ancianos. Rev Esp de Geriat y Geront 1988; 23:23-30.

8. Quinnet PPR. Haga una pregunta, salve una vida. USA: Instituto Quinnet; 1995.

9. Wasserman D. Suicide: an unnecessary death. London: Edited by Martin Dunitz; 2001.

10. World Health Organization (WHO). Safer Access to pesticide community interventions. Genève: WHO; 2006.

11. World Health Organization (WHO). Preventing suicide: A resource for primary health care workers. Genève: WHO; 2000.

12. World Health Organization (WHO). Preventing suicide: A resource for teachers and other school staff. Genève: WHO; 2000.

13. World Health Organization (WHO). Preventing suicide: a resource for general physicians. Genève: WHO; 2000.
14. World Health Organization (WHO). Preventing suicide: A resource for counsellors. Genève: WHO; 2006.

15. World Health Organization (WHO). Preventing suicide: A resource at work. Genève: WHO; 2006.

16. World Health Organization (WHO). Suicide and Suicide prevention in Asia. Genève: WHO; 2008.

17. Beck A. Resnik H, Lettieri D. The prediction of suicide. Pennsylvania: The Charles Press Publisher; 1974.

18. Conwell Y. Elder abuse. A risk factor for suicide? Crisis 1995; 16(3):104-105.

19. Eldrid J. Caring for the suicidal. London: Editorial Constable; 1993.

20. Sarro BCC. Los suicidios. Barcelona: Editorial Martínez Roca; 1991.

21. Sarro BCC. Suicidios y Supervivientes. Vertex Rev. Arg. De Psiq. 1996; 7:25-31.

22. Seguin M, Lesague AKM. History of early loss among a group of suicide survivors. Crisis 1995; 16(3):121125.

23. Shneidman E. The suicidal mind. Suicide as a Psychache Befriending. Worldwide Journal 1997; 3:5-6.

24. Correa H, Pérez B. El suicidio: una muerte evitable. Editora Atheneu: Brasil; 2006.

Artigo apresentado em 02/03/2012

Aprovado em 07/04/2012 